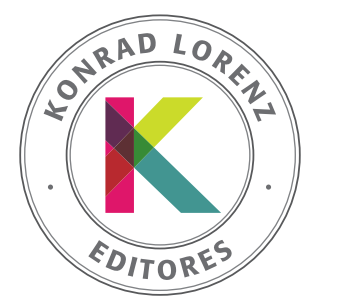

SUMA PSICOLÓGICA

http://editorial.konradlorenz.edu.co/suma-psicologica.html

\title{
Modelo de violencia en relaciones de pareja en adolescentes colombianos
}

\author{
María Margarita Rozo-Sánchez, Jaime Humberto Moreno-Méndez, \\ Sandra Jimena Perdomo-Escobar y Bertha Lucía Avendaño-Prieto
}

Universidad Católica de Colombia, Bogotá, Colombia

Recibido el 6 de Marzo de 2019; aceptado el 23 de Mayo de 2019

\author{
PALABRAS CLAVE \\ violencia, \\ adolescencia, \\ noviazgo, \\ victimización, \\ adaptación
}

\begin{abstract}
Resumen El presente estudio tuvo como objetivo elaborar un modelo sobre la asociación de los problemas de adaptación con la agresión y la victimización de la violencia en las relaciones de parejas adolescentes colombianas. Se tomó una muestra de 599 adolescentes con edades entre 13 y 19 años, escolarizados en instituciones educativas públicas y privadas de Bogotá, Colombia. Se aplicaron el cuestionario del Sistema de Evaluación de la Conducta de Niños y Adolescentes, versión autoinforme, y el inventario de conflicto en las relaciones en el noviazgo adolescente. Para el análisis de los datos, se utilizó la técnica de ecuaciones estructurales. Se presentan dos modelos determinados diferencialmente por la variable sexo: en los hombres los problemas de adaptación se relacionan con la agresión y en las mujeres se asocian con la victimización, hallazgos que ofrecen lineamientos para la prevención e intervención de la violencia de pareja en adolescentes.
\end{abstract}

(c) 2019 Fundación Universitaria Konrad Lorenz. Este es un artículo Open Access bajo la licencia CC BY-NC-ND (http://creativecommons.org/licenses/bync-nd/4.0/).

\section{Model of violence in the relationships of Colombian adolescent couples}

Abstract The present study aimed to develop a model of the association of adjustment problems with aggression and victimization of violence in Colombian adolescent couple relationships. A sample was taken of 599 adolescents with ages between 13 and 19 years, schooled in public and private educational institutions of Bogota, Colombia. The Children and Adolescents Behavior Assessment System Questionnaire -version of Self-Report and the Conflict Inventory in Relationships in Adolescent Dating was applied. For the analysis of the data, a model of structural equations was used. Two models determined differentially by the sex variable are presented; In men, adjustment problems are related to aggression and in women they are associated with victimization, findings that offer guidelines for the prevention and intervention of partner violence in adolescents. (c) 2019 Fundación Universitaria Konrad Lorenz. This is an open access article under the CC BY-NCND license (http://creativecommons.org/licenses/bync-nd/4.0/).

\footnotetext{
* Autor para correspondencia.

Correo electrónico: jhmoreno@ucatolica.edu.co.
} 
La violencia de pareja ha sido un problema frecuentemente abordado en diferentes estudios dada su alta prevalencia e incidencia (Heinze et al., 2018; Mohammad-Alizadeh-Charandabi, Bahrami-vazir, Kamalifard \& Mirghafourvand, 2016; Wincentak, Connolly \& Card, 2017); sin embargo, las investigaciones en adolescentes relacionadas con problemas de adaptación, agresión y victimización de la violencia de pareja no han sido suficientes, particularmente en el contexto colombiano.

En el noviazgo adolescente, se empiezan a configurar patrones de relación que pueden ser sanos o disfuncionales, que, unidos a variables individuales y contextuales, terminen en conductas violentas en la pareja (Oudererk, Blachman \& Mulford, 2014).

La violencia de pareja adolescente se define como el intento de dañar o controlar física, psicológica, económica, emocional y sexualmente a la pareja. Puede ocurrir de forma aislada o continua, entre parejas heterosexuales o del mismo sexo, dirigida hacia exparejas e inclusive cara a cara o de manera online (Rey-Anacona, 2009; Rodríguez-Domínguez, Durán-Segura \& Martínez-Pecino, 2018; Sugg, 2015).

La violencia en parejas heterosexuales es unidireccional cuando uno de los integrantes ha ejercido malos tratos o bidireccional cuando ambos miembros de la pareja actúen al mismo tiempo como víctima y agresor (Arnoso, Ibabe, Arnoso \& Elgorriaga, 2017), aunque la mayoría de las agresiones físicas durante el noviazgo son bidireccionales (Rey-Anacona, 2013).

En cuanto a la prevalencia de violencia de pareja entre adolescentes, se ha reportado un $20 \%$ de violencia física y un $9 \%$ de violencia sexual. El $35.8 \%$ han sido víctimas y el $33 \%$ maltratadores. Por sexo, las mujeres recurren más a amenazas e insultos y en ocasiones agresiones físicas, mientras que los hombres ejercen más la violencia sexual (Pazos, Oliva \& Gómez, 2014; Shorey, Fite, Cohen, Stuart \& Temple, 2018; Wincentak et al., 2017).

Se ha encontrado que el deterioro en la relación de pareja está relacionado con ser víctima de agresión verbal-emocional, mientras que el deterioro físico está asociado con haber sido víctima de agresión física (Fernández-Fuertes \& Fuertes, 2010; Rubio-Garay, Carrasco, Amor \& LópezGonzález, 2015).

La evidencia previa ha dado cuenta de varios factores de riesgo de violencia de pareja adolescente, que se ha relacionado con la inadaptación del propio adolescente (Temple, Shorey, Fite, Stuart \& Le 2013). La búsqueda de sensaciones que, en los adolescentes permite evidenciar el consumo de alcohol y marihuana o estar involucrados en vandalismo, puede generar mayor probabilidad de ser víctima de violencia en el noviazgo (Van Ouytse, Ponnet \& Walrave, 2017). Ho, Barry y Koeppel (2018) hallaron que la impulsividad puede convertirse en factor de riesgo para ejercer la violencia.

Por otro lado, se ha encontrado que, cuando las mujeres tratan de suprimir el enojo en las relaciones de pareja, presentan trastornos somatomorfos (Koh, Kim, Kim \& Park, 2005; Liu, Cohen, Schulz \& Waldinger, 2011). En los hombres, hay vulnerabilidad a presentar síntomas somáticos debido a un aumento en la producción de testosterona, al estar más expuesto a enojos frecuentes y prolongados (Herrero, Gadea, Rodríguez-Alarcón, Espert \& Salvador, 2010). La evidencia ha mostrado elevados niveles de cortisol resultado de estrés crónico en personas que experimentaron violencia de pareja (Yim \& Kofman, 2018), así como reactividad fisiológica incrementada y dificultades en regulación emocional (Davies, Sturge-Apple, Cicchetti, Manning \& Zale, 2009).

En otras investigaciones, se ha hallado una asociación entre síntomas emocionales de ansiedad y depresión con violencia sobre la pareja (Ruiz-Pérez, Rodríguez-Barranco, Cervilla \& Ricci-Cabello, 2018; Yu, Peplerb, Van de Bongardt, Josephsond \& Connolly, 2018), así como entre depresión y victimización (Judd, Schettler, Coryell, Akiskal \& Fiedorowicz, 2013; Øverup, Hadden, Knee \& Rodríguez, 2017; Van Ouytse et al., 2017).

Una variable que ha sido poco estudiada en el contexto de la violencia de pareja es el desajuste escolar. Se ha reportado que la exposición a la violencia doméstica de los padres se asocia con aislamiento de los hijos en el entorno escolar y dificultades en las relaciones de pareja (Hungerford, Wait, Fritz \& Clements, 2012; Namy et al., 2017).

El insuficiente apoyo social se constituye en un factor de riesgo para que los adolescentes de ambos sexos evidencien violencia hacia su pareja (Hungerford, Wait, Fritz \& Clements, 2012; Ruiz-Pérez et al., 2018; Temple et al., 2016).

En cuanto al desajuste personal, la evidencia ha reportado una relación entre violencia física, verbal, sexual, amenazas y relacional, con la dimensión física y emocional del autoconcepto (Lila, Gracia \& Herrero, 2012; Penado-Abilleira \& Rodicio-García, 2017; Pflieger \& Vazsonyi, 2006; Rubio-Garay et al., 2015). También, en adolescentes con menor confianza en sí mismos para resolver conflictos en la relación (Fernández-González, Calvete, Orue \& Echezarraga, 2018), tener consumo de alcohol y drogas (Calvete, Gámez-Guadix, Fernández-Gonzalez, Orue \& Borrajo, 2018; Fagan \& Wright, 2011; Latzmana, VivoloKantorb, Clinton-Sherroda, Casanuevaa \& Carr, 2017; Pflieger \& Vazsonyi, 2006; Roberts, Gilman, Fitzmaurice, Decker \& Koenen, 2010; Tenkorang \& Owusu, 2018), y problemas de comportamiento externalizante e internalizante (GrahamBermann, Griber, Howell \& Girz, 2009; Vu, Jouriles, McDonald \& Rosenfield, 2016).

Finalmente, se ha identificado que los adolescentes con antecedentes de maltrato infantil y estilos de apego evitativo pueden tener un mayor riesgo de participación en violencia en el noviazgo (Stovera, Choib \& Mayesa, 2018).

Por otro lado, se han reportado varios factores protectores de la violencia de pareja y promotores de la adaptación psicológica en adolescentes, tales como altos niveles de apoyo íntimo percibido proporcionado por la pareja, amigos, parientes cercanos y organizaciones comunitarias (Lila, Gracia \& Murgui, 2013). Un estilo parental basado en la sensibilidad y en la calidez puede contribuir favorablemente en la respuesta emocional del adolescente al conflicto dado que posibilita reducir la amenaza percibida, así como incrementar la eficacia para afrontar y modular su expresividad emocional (Bernier, Beauchamp, Carlson \& Lalonde, 2015; Cummings, Koss \& Davies, 2015; Lamela, Jongenelen, Pinto \& Levendosky, 2018).

La evidencia preliminar justifica la importancia de establecer un modelo sobre la asociación de los problemas de adaptación con la agresión y la victimización de la violencia en las relaciones de parejas adolescentes colombianas. A partir de los anterior, se plantearon las siguientes hipótesis 
reflejadas en el modelo propuesto en la figura 1. (H1) Los problemas de adaptación estarán relacionados directa y positivamente con la agresión de los hombres en la violencia de pareja. ( $\mathrm{H} 2)$ Los problemas de adaptación estarán relacionados directa y positivamente con la victimización de las mujeres en la violencia de pareja.

\section{Método}

\section{Participantes}

A partir del dato poblacional de adolescentes colombianos que corresponde a un total de 4346059 (Departamento Administrativo Nacional de Estadística [DANE], 2010), con un nivel de confianza del $95 \%$ y un margen de error del $5 \%$, mediante el programa estadístico GPower, se necesitaban 400 participantes, no obstante, se obtuvo una muestra total de 599 adolescentes de los que 327 eran del sexo femenino $(M=16.9 ; D E=1.45)$ y 272 de sexo masculino $(M=17.35$; $D E=1.35)$. El muestreo fue por conveniencia, se invitaron nueve instituciones educativas de diferente tipo (pública y privada), jornada (mañana, tarde y noche) y estrato socioeconómico, seis de las que aceptaron participar voluntariamente en la investigación. Los criterios de inclusión de los adolescentes fueron (a) haber tenido o tener una relación amorosa de al menos un mes de duración, (b) tener entre 13 y 19 años, (c) ser soltero y (d) contar con la autorización de la institución educativa para realizar el estudio, con el consentimiento escrito de los padres y el asentimiento del adolescente. Se obtuvo representación de estratos socioeconómicos bajos, medios y altos. Las características sociodemográficas más representativas fueron las siguientes. En hombres, el $40.4 \%$ pertenecían al estrato socioeconómico bajo, el $54.8 \%$ cursaban último grado de bachillerato, el $93.8 \%$ se identificaron como heterosexuales, el $20 \%$ reportaron haber tenido dos parejas y el $5.9 \%$ tenían un mes de duración con la relación actual. En mujeres, el $43.4 \%$ pertenecían al estrato socioeconómico bajo, el $38.2 \%$ cursaban último grado de bachillerato, el $92 \%$ se identificaron como heterosexuales, el $36 \%$ reportaron haber tenido dos parejas y el $4.9 \%$ tenían dos meses de duración con la relación actual.

\section{Instrumentos}

Sistema de evaluación de la conducta en niños y adolescentes, versión de autoinforme (BASC-S3). El BASC es un sistema de evaluación multimétodo que permite valorar a los niños y adolescentes a través del reporte de sus padres y profesores, y el autoinforme (González, Fernández, Pérez \& Santamaría, 2004). Se utilizó la versión de autoinforme que consta de 185 ítems e incluye las siguientes escalas: actitud negativa hacia el colegio y hacia los profesores, búsqueda de sensaciones, atipicidad, locus de control, somatización, estrés social, ansiedad, depresión, sentido de incapacidad, autoestima, confianza en sí mismo, relaciones interpersonales y relaciones con los padres. También permite obtener tres índices globales: desajuste escolar, desajuste clínico, ajuste personal e índice de síntomas emocionales. La confiabilidad de las escalas se ubica entre 0.70 y 0.80 . Con población colombiana infantil y adolescente, se utilizó para predecir problemas emocionales afectivos; los resultados reportan evidencia de validez (Hewitt, 2015).

\section{Conflict in Adolescent Dating Relationships Inven- tory (CADRI), versión española.}

Fue creado originalmente por Wolfe, Crooks, Lee, McIntyre-Smith \& Jaffe (2003). Evalúa comportamientos relacionados con victimización y perpetración de violencia física, verbal-emocional, relacional, sexual y amenazas en parejas adolescentes en los últimos doce meses (Fernández-Fuertes et al., 2006).

Tiene 35 díadas de ítems en escala Likert con cuatro opciones de respuesta y cinco factores: física (e. g., "Le lancé algún objeto"), sexual (e. g., "Le besé cuando él/ella no quería”), verbal (e. g., “Le culpé por el problema”), relacional (e. g., "Extendí rumores falsos sobre él/ella"), amenazas (e. g., "Amenacé con herirle").

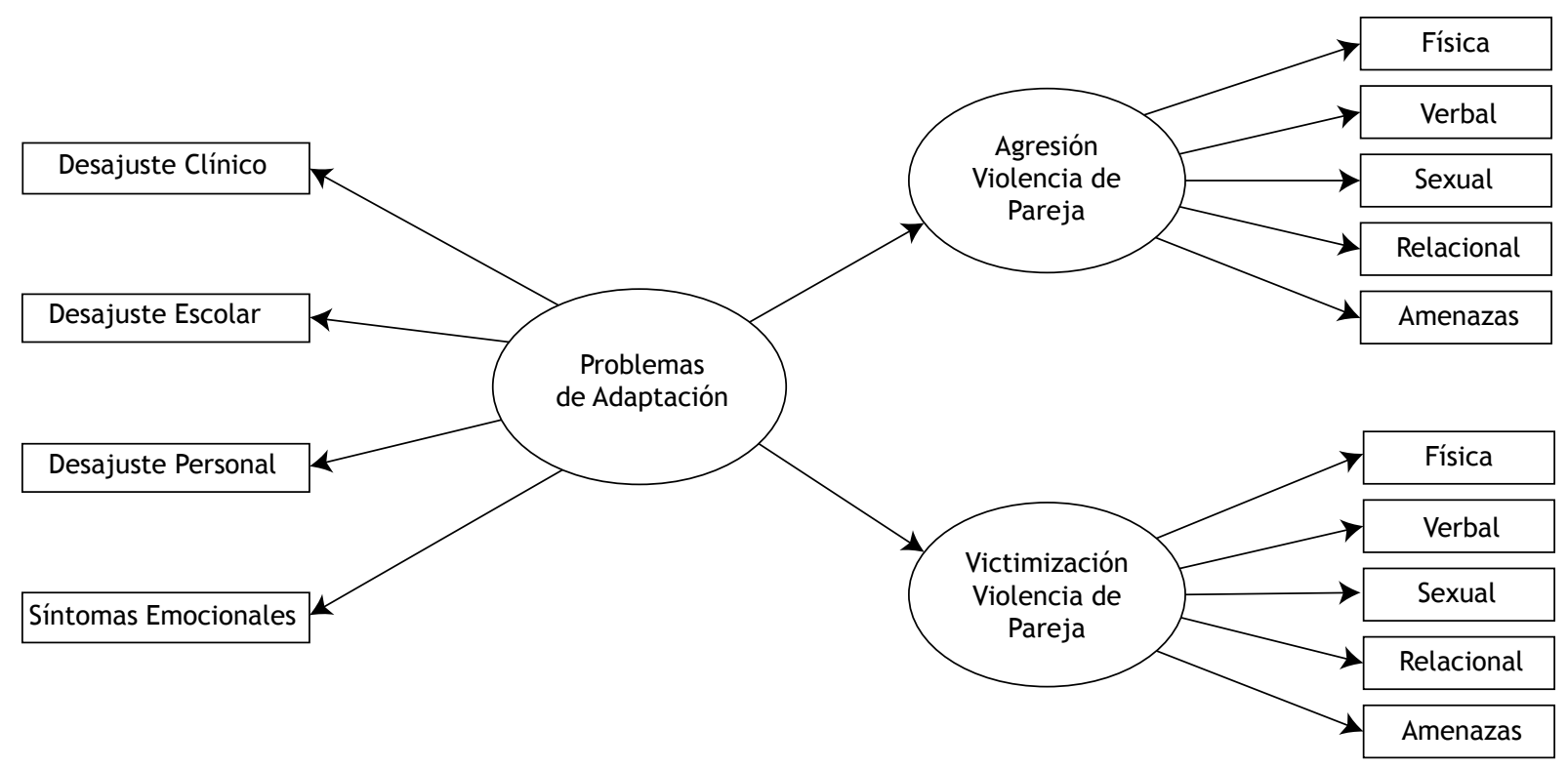

Figura 1. Modelo propuesto de los problemas de adaptación y la violencia de pareja adolescente. 
Los índices de consistencia interna de las subescalas reportados por los autores oscilaron entre .51 y .79 con un valor alfa de .86 para todo el cuestionario. En el presente estudio, los alfas oscilaron entre .69 y .90 con un alfa de .93 para todo el instrumento. El análisis factorial exploratorio evidenció una estructura de seis factores que explican el $54.23 \%$ de la varianza, aunque un análisis forzado mostró que cinco factores podrían explicar al menos el $50 \%$ de esta. Este instrumento se utilizó en esta investigación por las siguientes razones: (a) se cuenta con una versión en español de este que se adecúa al español hablado en Colombia, (b) informa sobre la victimización y la perpetración, y (c) contiene conductas de maltrato que son comunes en parejas jóvenes.

\section{Procedimiento}

En el primer semestre de 2018, se obtuvo la autorización de las instituciones educativas para aplicar los instrumentos en horas de clase. Previo consentimiento de los padres de familia y asentimiento de los adolescentes, se aplicaron los instrumentos en físico, con un código, de manera grupal, durante cuarenta y cinco minutos aproximadamente. Los datos fueron procesados con el software SPSS versión 23. A las instituciones educativas se les presentaron los resultados del estudio y a los adolescentes les fueron entregadas unas cartillas psicoeducativas para el fortalecimiento de sus relaciones de pareja.

\section{Análisis de datos}

Se utilizó un modelo de ecuaciones estructurales, previo cumplimiento de los supuestos requeridos para el uso de esta técnica. Para el análisis de bondad de ajuste del modelo, se siguieron las indicaciones señaladas en la literatura (Pilatti, Godoy \& Brussino, 2012), se utilizó la razón de chi cuadrado sobre los grados de libertad CMIN/DF, los índices CFI y GFI, el índice RMSEA, el índice no normalizado de ajuste o Tucker-Lewis (TLI), y el Incremental Fit Index (IFI) (Leal-Costa, Tirado-González, Van-der Hofstadt Román \& Rodríguez-Marín, 2016). El modelo propuesto implica la relación entre los variables problema de adaptación y agresión y victimización de la violencia en relaciones de pareja adolescente.

\section{Resultados}

El análisis de la relación entre las variables se llevó a cabo con el modelo de ecuaciones estructurales. Dado que las variables no se ajustaron a una distribución normal, se empleó el método de distribución asintóticamente libre (ADF, por sus siglas en inglés), que es utilizado cuando las variables del modelo son categóricas, y con un $n>250$ (Lara, 2014; Manzano, 2017). Además, se correlacionaron todas las variables y sus dimensiones con el coeficiente Rho de Spearman, que oscilaron entre .41 y .95 , todas significativas al $p<.001$.

Se encontró que en los hombres el modelo establece relaciones sobre la violencia. Como se aprecia en la tabla 1, el puntaje del CMIN/DF fue de 1.18, valores inferiores a tres que indican un buen ajuste. El índice de bondad de ajuste global (GFI.97) y el índice de ajuste comparativo (CFI.97), que varían entre 0 y 1 , en los que 0 indica ausencia de ajuste y 1 ajuste óptimo. El índice de Tucker-Lewis (TLI.95) y el Incremental Fit Index (IFI.97), considerados aceptables por encima de .90 , y el error cuadrado de aproximación a las raíces medias RMSEA =.03[.00;.07], que es considerado excelente cuando sus valores son de .05 o inferiores y aceptable en el rango de .05 y .08 (Hu \& Bentler, 1995).

El modelo presentado en la figura 2 plantea que el índice de síntomas emocionales, conformado por indicadores de estrés y sentido de incapacidad, así como el desajuste escolar, conformado por actitud negativa hacia el colegio y hacia los profesores, están asociados con la agresión hacia su pareja en hombres adolescentes.

Al interpretar la magnitud y el signo de los parámetros estimados, los resultados constatan que el estrés y el sentido de incapacidad percibida, que conforman el factor denominado síntomas emocionales, se correlacionan con el factor denominado desajuste escolar en .44. $(p=.00)$. Asimismo, el desajuste escolar está relacionado con la agresión en .19 $(p=.01)$. Los síntomas emocionales tienen una asociación con la agresión de -.21 ( $p=.01)$. La varianza explicada de cada una de las variables que conforman el factor agresión osciló entre .30 (violencia sexual) y .51 (amenazas).

En cuanto a los resultados con las mujeres, el modelo da cuenta de la victimización. En la tabla 2, se presentan los estadísticos de bondad de ajuste utilizados (CMIN/DF = 1.20), (GFI.98), (CFI.96), (TLI.94), (IFI.97) y RMSEA=.03[.00;.06]. Todos indican un buen ajuste del modelo.

En la figura 3, se muestra que el desajuste escolar, conformado por actitud negativa hacia el colegio y hacia los profesores, así como el índice de síntomas emocionales, conformado por estrés social y sentido de incapacidad, están asociados significativamente con la victimización y aportan al modelo en $-0.31(p=.02)$ y $.27(p=.01)$, respectivamente. Los resultados constatan que la covarianza o variación conjunta entre los síntomas emocionales con los factores de desajuste escolar es significativa e igual a .66

Tabla 1. Estadísticos utilizados para el modelo estructural de la agresión en hombres

\begin{tabular}{ccccccc}
\hline Indicadores & CMIN/DF & GFI & CFI & TLI & IFI & RMSEA \\
\hline Puntajes & 1.18 & .97 & .97 & .95 & .97 & $.03[.00 ; .07]$ \\
\hline
\end{tabular}

CMIN/DF = Razón del chi cuadrado sobre los grados de libertad GFI = Índice global de bondad de ajuste; CFI = Índice de ajuste comparativo; TLI = Índice de Trucker-Lewis; AIC = criterio de información de Akaike; IFI = Índice de ajuste incremental; RMSEA = Error cuadrático medio.

Tabla 2. Estadísticos utilizados para el modelo estructural de victimización en mujeres

\begin{tabular}{ccccccc}
\hline Indicadores & CMIN/DF & GFI & CFI & TLI & IFI & RMSEA \\
\hline Puntajes & 1.20 & .98 & .96 & .94 & .97 & $.03[.00 ; .06]$ \\
\hline
\end{tabular}

CMIN/DF = Razón del chi cuadrado sobre los grados de libertad; GFI = Índice global de bondad de ajuste; CFI = Índice de ajuste comparativo; TLI = Índice de Trucker-Lewis; AIC = criterio de información de Akaike; IFI = Índice de ajuste incremental; RMSEA = Error cuadrático medio. 


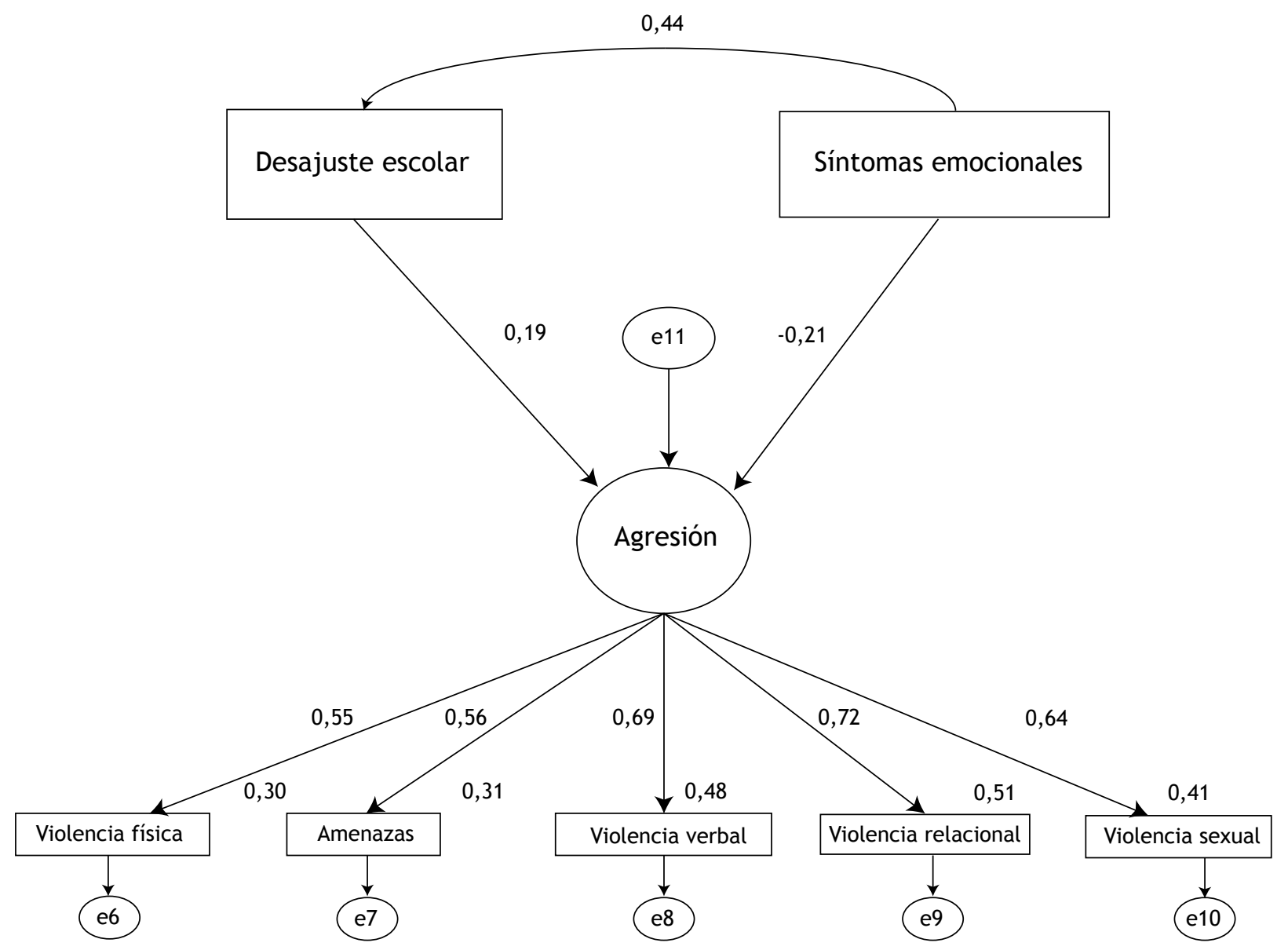

Figura 2. Modelo estructural de los problemas de adaptación y la agresión de violencia de pareja en hombres adolescentes

$(p=.00)$. La varianza explicada de cada una de las variables que conforman el factor victimización osciló entre .19 (amenazas) y .54 (violencia verbal).

\section{Discusión}

El objetivo del presente estudio consistió en establecer un modelo asociativo de los problemas de adaptación con la agresión y la victimización de la violencia de parejas adolescentes colombianas. En nuestros resultados, se encontraron dos modelos.

La hipótesis 1, referida a la relación entre los problemas de adaptación y agresión de los hombres en la violencia de pareja, fue parcialmente confirmada, dado que en el modelo únicamente el desajuste escolar y los síntomas emocionales estuvieron asociados a la agresión hacia sus parejas, mientras que el desajuste personal y clínico no estuvieron correlacionados. Asimismo, el estrés y el sentido de incapacidad fueron las variables que mejor estuvieron relacionadas con el desajuste escolar. Desde la perspectiva del ajuste psicológico, coincide con el estudio de Lila et al. (2013), que mostró que el apoyo social y los eventos estresantes están vinculados con el ajuste psicológico. Igualmente, el estrés se encuentra asociado con dificultades en regulación emocional que podrían tener un impacto en el hombre en la expresión de violencia hacia su pareja (Davies et al., 2009).
Lo anterior implica que el estrés, el sentido de incapacidad y la actitud negativa hacia el colegio y hacia los profesores en su conjunto se encuentran asociados a violencia verbal, relacional, sexual, física y amenazas hacia sus parejas por parte de los hombres, lo que es consistente con estudios previos en los que se ha informado sobre las diferentes formas de manifestación de la violencia de pareja que evidencian los adolescentes maltratadores (Rey-Anacona, 2009; Rodríguez-Domínguez et al., 2018; Sugg, 2015).

Las dificultades de ajuste de los adolescentes que se encontraron en el presente estudio probablemente se encuentran relacionados con el tiempo y la duración de la exposición a la violencia de pareja (DeBoard-Lucas \& Grych, 2011). Acorde con las medidas del BASC empleadas en este estudio, estos hallazgos indicarían que los adolescentes que se perciben con falta de confianza en sí mismos, con dificultades para perseverar en sus metas y con insatisfacción con el ambiente escolar y con sus profesores tienden a tener mayor tendencia a violentar a sus parejas.

La hipótesis 2, referida a la relación directa y positiva entre los problemas de adaptación y la victimización de violencia de pareja en mujeres, fue parcialmente confirmada debido a que se encontró una mayor victimización en el sexo femenino probablemente en respuesta a un aprendizaje por observación de modelos de violencia de pareja (Forkea et al., 2018; Heinze et al., 2018; Shorey et al., 2018). 


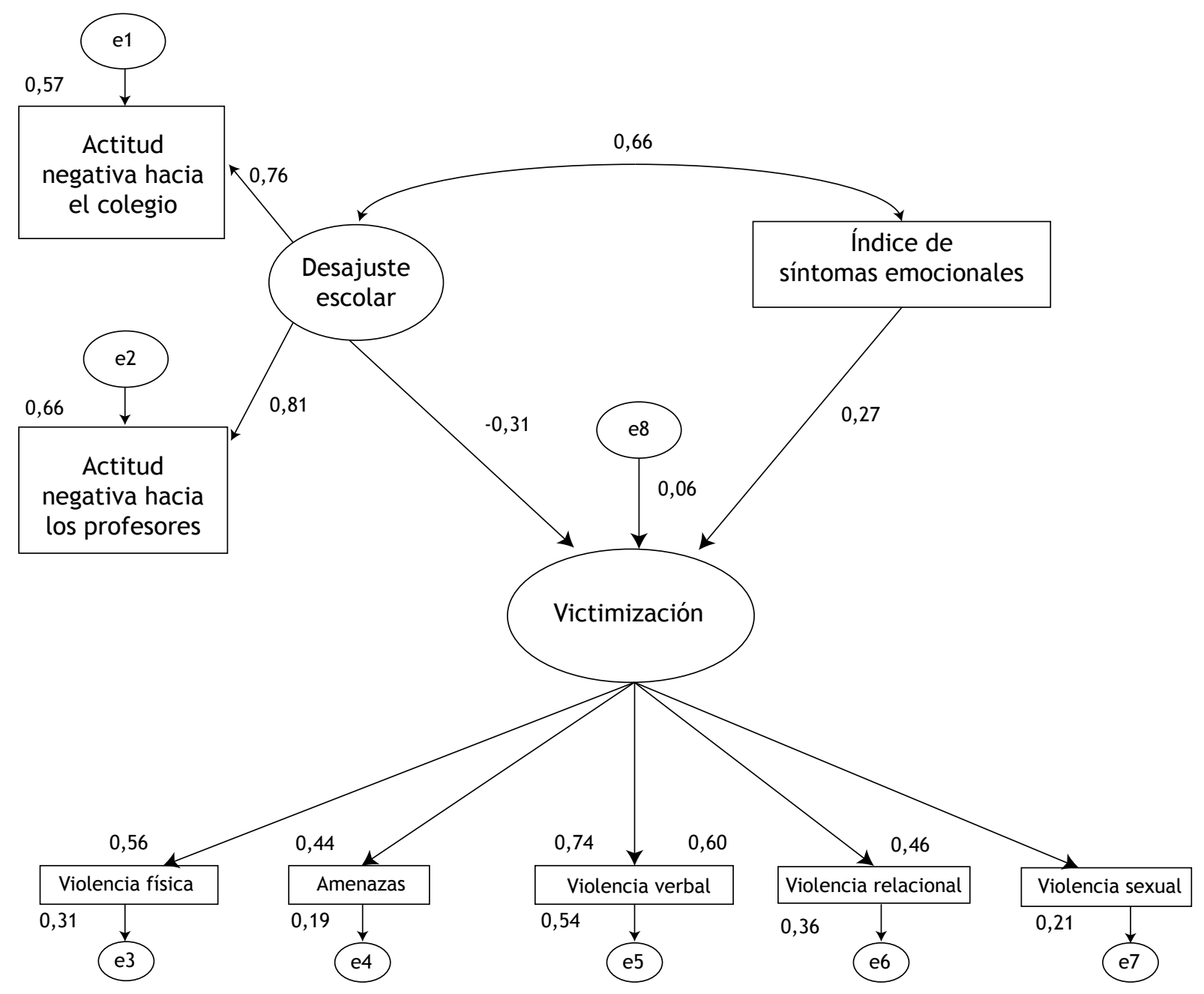

Figura 3. Modelo estructural de los problemas de adaptación y la victimización de violencia de pareja en mujeres adolescentes.

Por otro lado, el desajuste personal y clínico no se asociaron con victimización, mientras que el desajuste escolar y los síntomas emocionales mostraron una correlación con la victimización. Esto podría sugerir que una actitud desfavorable hacia el colegio podría constituirse en una fuente de victimización de la violencia de pareja (Puente-Martínez, Ubillos-Landa, Echeburúa \& Páez-Rovira, 2016). Resultados que indicarían que las adolescentes que no se encuentran satisfechas en el entorno escolar y perciben que no cuentan con el apoyo de profesores estarían en riesgo de no buscar apoyo en este ambiente para prevenir su victimización.

Otra de las variables relacionadas con la victimización en las mujeres fue el estrés social, lo que podría significar que la percepción de estrés crónico incrementa el riesgo a ser víctima de violencia en las relaciones de pareja (Yim \& Kofman, 2018). También podría decirse que las adolescentes que experimentan estrés social evidencian sensaciones de tensión por falta de recursos de afrontamiento y de apoyo, especialmente de amigos y familiares, así como dificultades en regulación emocional (Davies et al., 2009), lo que las hace más vulnerables a la violencia por parte de sus parejas. En otro sentido, se ha reportado que la violencia de pareja puede generar mayor estrés en las víctimas. Estas dos direcciones podrían suponer las características del ciclo de la violencia de pareja (Yim \& Kofman, 2018).

El sentido de incapacidad también estuvo asociado a la victimización en las adolescentes. Este hallazgo es consistente con evidencia científica previa que subraya que las creencias de bajo control percibido sobre el ambiente y su futuro favorecen la vulneración de los derechos en las adolescentes (Musci, Bettencourt, Rabinowitz, lalongo \& Lambert, 2018). Cuando las adolescentes perciben que no pueden defender sus derechos al ser violentadas por sus parejas, favorece su victimización.

A diferencia de los dos modelos encontrados, el estudio realizado por Vu et al. (2016) no encontró una asociación según sexo entre los problemas de ajuste y la violencia de pareja. Además, Cortés-Ayala et al. (2015) reportan que las adolescentes evidencian mayor maltrato físico y menos victimización en el noviazgo, mientras que los hombres tienen una percepción de victimización, especialmente de tipo físico, emocional e instrumental, aspectos que requerirían mayor estudio.

En este orden de ideas, una fortaleza de esta investigación ha sido presentar a través de dos modelos los diferentes factores asociados con el maltrato y la victimización de la violencia en las relaciones de pareja en una muestra de adolescentes colombianos. Estos hallazgos se constitu- 
yen en un insumo para orientar procesos de prevención e intervención de este fenómeno en población adolescente (Stovera, Choib \& Mayesa, 2018; Yu et al., 2018).

Una limitación del estudio fue no haber incluido muestra clínica que permitiera contrastar los resultados con la muestra analizada. Dado que es un estudio transversal, no se pueden establecer explicaciones causales que permitan dar cuenta con mayor precisión del fenómeno de la violencia de pareja adolescente a partir de los problemas de adaptación. Por lo anterior, se sugieren llevar a cabo estudios longitudinales que incluyan muestras clínicas y estudios de carácter experimental y transcultural que den cuenta de variables predictivas de la violencia de pareja adolescente.

Los modelos probados en el presente estudio permiten sugerir para una futura investigación evaluar la eficacia de un programa de prevención de la violencia de pareja adolescente, que incluya como componentes para el trabajo con hombres el manejo del estrés y el fortalecimiento de la autoestima y de las relaciones con el colegio. En mujeres, estrategias de fortalecimiento de comunicación y apoyo desde el ambiente escolar para prevenir su victimización. Igualmente, desarrollar estrategias de afrontamiento para el manejo del estrés social y fortalecimiento de la percepción de capacidad para defender sus derechos a no ser violentadas por su parejas.

\section{Referencias}

Arnoso, A., Ibabe, I., Arnoso, M. \& Elgorriaga, E. (2017). El sexismo como predictor de la violencia de pareja en un contexto multicultural. Anuario de Psicología Jurídica, 27(1), 9-20. http:// dx.doi.org/10.1016/j.apj.2017.02.001

Bernier, A., Beauchamp, M. H., Carlson, S. M. \& Lalonde, G. (2015). A secure base from which to regulate: Attachment security in toddlerhood as a predictor of executive functioning at school entry. Developmental Psychology, 51(9), 1177-1189. http://dx. doi.org/10.1037/dev0000032

Calvete, E., Gámez-Guadix, M., Fernández-Gonzalez, L., Orue, I. \& Borrajo, E. (2018). Maladaptive schemas as mediators of the relationship between previous victimizations in the family and dating violence victimization in adolescents. Child Abuse \& Neglect, 81, 161-169. http://dx.doi.org/10.1016/j.chiabu.2018.04.028

Cortés-Ayala, L., Flores Galaz, M., Bringas Molleda, C., Rodríguez-Franco, L., López-Cepero Borrego, J. \& Rodríguez Díaz, F. J. (2015). Relación de maltrato en el noviazgo de jóvenes mexicanos: análisis diferencial por sexo y nivel de estudios. Terapia Psicológica, 33(1), 5-12. http://dx.doi.org/10.4067/S071848082015000100001

Cummings, E. M., Koss, K. J. \& Davies, P. T. (2015). Prospective relations between family conflict and adolescent maladjustment: Security in the family system as a mediating process. Journal of Abnormal Child Psychology, 43(3), 503-515. http://dx.doi. org/10.1007/s10802-014-9926-1

Davies, P. T., Sturge-Apple, M. L., Cicchetti, D., Manning, L. G. \& Zale, E. (2009). Children's patterns of emotional reactivity to conflict as explanatory mechanisms in links between interpartner aggression and child physiological functioning. Journal of Child Psychology and Psychiatry, 50(11), 1384-1391. http:// dx.doi.org/10.1111/j.1469-7610.2009.02154.x

Departamento Administrativo Nacional de Estadística. (2010). Informe de gestión 2010. Bogotá, Colombia: Autor. Recuperado de https://www.dane.gov.co/files/control_participacion/rendicion_cuentas/informe_gestionfinan_dane10.pdf
DeBoard-Lucas, R. L. \& Grych, J. H. (2011). The effects of intimate partner violence on school-age children. En S. A. Graham-Bermann \& A. A. Levendosky (Eds.). How intimate partner violence affects children: Developmental research, case studies, and evidence-based intervention (pp. 55-77). Washington, DC, EE. UU.: American Psychological Association.

Fagan, A. A. \& Wright, E. M. (2011). Gender differences in the effects of exposure to intimate partner violence on adolescent violence and drug use. Child Abuse \& Neglect, 35(7), 543-550. http://dx.doi.org/10.1016/j.chiabu.2011.05.001

Fernández-Fuertes, A. A. \& Fuertes, A. (2010). Physical and psychological aggression in dating relationships of Spanish adolescents: Motives and consequences. Child Abuse \& Neglect, 34(3), 183-191. http://dx.doi.org/10.1016/j.chiabu.2010.01.002

Fernández-Fuertes, A. A., Fuertes, A. \& Pulido, R. F. (2006). Evaluación de la violencia en las relaciones de pareja de los adolescentes: validación del Conflict in Adolescent Dating Relationships Inventory (CADRI)-versión española. International Journal of Clinical and Health Psychology, 6(2), 339-358.

Fernández-González, L., Calvete, E., Orue, I. \& Echezarraga, A. (2018). The role of emotional intelligence in the maintenance of adolescent dating violence perpetration. Personality and Individual Differences, 127, 68-73. http://dx.doi.org/10.1016/j. paid.2018.01.038

Forkea, C. M., Myersb, R. K., Feinb, J. A., Catallozzie, M., Localioa, A. R., Wiebea, D. J. \& Grissoh, J. A. (2018). Witnessing intimate partner violence as a child: How boys and girls model their parents' behaviors in adolescence. Child Abuse \& Neglect, 84, 241-252. http://dx.doi.org/10.1016/j.chiabu.2018.07.031

González, J., Fernández, S., Pérez E. \& Santamaría, P. (2004). Adaptación española del sistema de evaluación de la conducta en niños y adolescentes: BASC. Madrid, España: TEA.

Graham-Bermann, S. A., Gruber, G., Howell, K. H. \& Girz, L. (2009). Factors discriminating among profiles of resilience and psychopathology in children exposed to intimate partner violence (IPV). Child Abuse \& Neglect, 33(9), 648-660, http://dx. doi.org/10.1016/j.chiabu.2009.01.002

Heinze, J. E., Carter, P. M., Ngo, Q., Zimmerman, M. A., Walton, M. A. \& Cunningham, R. M. (2018). Patterns of partner and nonpartner violence among high-risk youth. Journal of Adolescent Health, 62(5), 598-604. http://dx.doi.org/10.1016/j. jadohealth.2017.11.304

Herrero, N., Gadea, M., Rodríguez-Alarcón, G., Espert, R. \& Salvador, A. (2010). What happens when we get angry? Hormonal, cardiovascular and asymmetrical brain responses. Hormones and Behavior, 57(3), 276-283. http://dx.doi.org/10.1016/j.yhbeh.2009.12.008.

Hewitt Ramírez, N. (2015). Predicción de los problemas de banda ancha y banda estrecha mediante la integración de los modelos transaccional, cognitive-social y de regulación emocional. (Tesis doctoral, Universidad de Granada, Granada, España). Recuperado de https://dialnet.unirioja.es/servlet/tesis?codigo $=56192$

Ho, B. C., Barry, B. A. \& Koeppel, J. A. (2018). Impulsivity in unaffected adolescent biological relatives of schizophrenia patients. Journal of Psychiatric Research, 97, 47-53. http://dx. doi.org/10.1016/j.jpsychires.2017.11.008

Hu, L. \& Bentler, P. (1995). Evaluating model fit. En R. Hoyle (Ed.), Structural equation modelling: Concepts, issues and applications (pp. 76-99). Thousand Oaks, EE. UU.: Sage.

Hungerford, A., Wait, S. K., Fritz, A. M. \& Clements, C. M. (2012). Exposure to intimate partner violence and children's psychological adjustment, cognitive functioning, and social competence: A review. Aggression and Violent Behavior, 17(4), 373-382. http://dx.doi.org/10.1016/j.avb.2012.04.002

Johnson, W. L, Manning, W. D., Giordano, P. C. \& Longmore, M. A. (2015). Relationship context and intimate partner violence from adolescence to young adulthood. Journal of Adolescent Health, 57(6), 631-636. http://dx.doi.org/10.1016/j.jadohealth.2015.08.014 
Judd, L. L., Schettler, P. J., Coryell, W., Akiskal, H. S. \& Fiedorowicz, J. G. (2013). Overt irritability/anger in unipolar major depressive episodes: Past and current characteristics and implications for long-term course. JAMA Psychiatry, 70(11), 11711180. http://dx.doi.org/10.1001/jamapsychiatry.2013.1957

Kimbera, M., Adhama, S., Gillb, S., McTavisha, J. \& MacMillan, H. L. (2018). The association between child exposure to intimate partner violence (IPV) and perpetration of IPV in adulthood-A systematic review. Child Abuse \& Neglect, 76, 273-286. http:// dx.doi.org/10.1016/j.chiabu.2017.11.007

Kitzmann, K. M., Gaylord, N. K., Holt, A. R. \& Kenny, E. D. (2003). Child witnesses to domestic violence: A meta-analytic review. Journal of Consulting and Clinical Psychology, 71(2), 339-352. http://dx.doi.org/10.1037/0022-006X.71.2.339

Koenen, K. C., Moffitt, T. E., Caspi, A., Taylor, A. \& Purcell, S. (2003). Domestic violence is associated with environmental suppression of IQ in young children. Development and Psychopathology, 15(2), 297-311 http://dx.doi.org/10.1017.S0954579403000166

Koh, K. B., Kim, D. K., Kim, S. Y. \& Park, J. K. (2005). The relation between anger expression, depression, and somatic symptoms in depressive disorders and somatoform disorders. Journal of Clinical Psychiatry, 66(4), 485-491. https://www.ncbi.nlm.nih. gov/pubmed/15816791

Lamela, D., Jongenelen, I., Pinto, R. \& Levendosky, A. (2018). Typologies of intimate partner violence-maternal parenting and children's externalizing problems: The moderating effect of the exposure to other forms of family violence. Child Abuse \& Neglect, 81, 60-73. http://dx.doi.org/10.1016/j.chiabu.2018.04.020

Lara Hormigo, A. (2014). Introducción a la ecuaciones estructurales en Amos y R. Recuperado de http://masteres.ugr.es/moea/pages/curso201314/tfm1314/tfm-septiembre1314/memoriamasterantonio_lara_hormigo/

Latzmana, N. E., Vivolo-Kantorb, A. M., Clinton-Sherroda, A. M, Casanuevaa, C. \& Carr, C. (2017). Children's exposure to intimate partner violence: A systematic review of measurement strategies. Aggression and Violent Behavior, 37, 220-235 http://dx.doi.org/10.1016/j.avb.2017.10.009

Leal-Costa, C., Tirado-González, S., Van-der Hofstadt Román, C. \& Rodríguez-Marín, J. (2016). Creación de la escala sobre habilidades de comunicación en profesionales de la salud, EHC-PS. Anales de Psicología, 32(1), 49-59. http://dx.doi.org/10.6018/ analesps.31.3.184701

Lila, M., Gracia, E. \& Herrero, J. (2012). Asunción de responsabilidad en hombres maltratadores: influencia de la autoestima, la personalidad narcisista y la personalidad antisocial. Revista Latinoamericana de Psicología, 44(2), 99-108.

Lila, M., Gracia, E. \& Murgui, S. (2013). Psychological adjustment and victim-blaming among intimate partner violence offenders: The role of social support and stressful life events. The European Journal of Psychology Applied to Legal Context, 5(2), 147-153. http://dx.doi.org/10.5093/ejpalc2013a4

Liu, L., Cohen, S., Schulz, M. S. \& Waldinger, R. J. (2011). Sources of somatization: Exploring the roles of insecurity in relationships and styles of anger experience and expression. Social Science \& Medicine, 73(9), 1436-1443. http://dx.doi.org/10.1016/j.socscimed.2011.07.034

Manzano, A. B. (2017). Metodología de investigación en educación médica, introducción a los modelos de ecuaciones estructurales. Investigación en Educación Médica, 7(25), 67-72. http:// dx.doi.org/10.1016/j.riem.2017.11.002

Merikangas, K. R., He, J., Burstein, M., Swanson, S. A., Avenevoli, S., Cui, L., Benjet, C., Georgiades, K. \& Swendsen, J. (2010). Lifetime prevalence of mental disorders in US adolescents: Results from the National Comorbidity Study-Adolescent Supplement (NCS-A). Journal of the American Academic of Child and Adolescent Psychiatry, 49(10), 980-989. http://dx.doi.org/10.1016/j.jaac.2010.05.017
Mohammad-Alizadeh-Charandabi, S., Bahrami-vazir, E. Kamalifard, M. \& Mirghafourvand, M. (2016). Intimate partner violence during the first pregnancy: A comparison between adolescents and adults in an urban area of Iran. Journal of Forensic and Legal Medicine, 43, 53-60. http://dx.doi.org/10.1016/j. jflm.2016.07.002

Musci, R. J., Bettencourt, A. F., Rabinowitz, J., lalongo, N. S. \& Lambert, S. F. (2018). Negative consequences associated with witnessing severe violent events: The role of control-related beliefs. Journal of Adolescent Health, 63(6), 739-744. http:// dx.doi.org/10.1016/j.jadohealth.2018.07.001

Namy, S., Carlson, C., Norcini Pala, A., Faris, D., Knight, L., Allen, E., Devries, K. \& Naker, D. (2017). Gender, violence and resilience among Ugandan adolescents. Child Abuse \& Neglect, 70, 303-314. http://dx.doi.org/10.1016/j.chiabu.2017.06.015

Oudererk, B., Blachman-Demner, D. \& Mulford, C. (2014). Teen dating violence: How peers can affect risk \& protective factors. Washington, DC, EE. UU.: U.S. Department of Justice.

Øverup, C. S., Hadden, B. W., Knee, C. R. \& Rodriguez, L. M. (2017). Self-determination theory and intimate partner violence (IPV): Assessment of relationship causality orientations as predictors of IPV perpetration. Journal of Research in Personality, 70, 139-155. http://dx.doi.org/10.1016/j.jrp.2017.07.002

Pazos Gómez, M., Oliva Delgado, A. \& Gómez, Á. H. (2014). Violencia en relaciones de pareja de jóvenes y adolescentes. Revista Latinoamericana de Psicología, 46(3), 148-159. http://dx.doi. org/10.1016/S0120-0534(14)70018-4

Penado-Abilleira, M. \& Rodicio-García, M. L. (2017). Análisis del autoconcepto en las víctimas de violencia de género entre adolescentes. Suma Psicológica, 24(2), 107-114. http://dx.doi. org/10.1016/j.sumpsi.2017.08.001

Pflieger, J. C. \& Vazsonyi, A. T. (2006). Parenting processes and dating violence: The mediating role of self-esteem in low-and high-SES adolescents. Journal of Adolescence, 29(4), 495-512. http://dx.doi.org/10.1016/j.adolescence.2005.10.002Medline

Pilatti, A., Godoy, J. C. \& Brussino, S. (2012). Análisis factorial confirmatorio del cuestionario de expectativas hacia el alcohol para adolescentes (CEA-A). Acta Colombiana de Psicología, 15(2), 11-20.

Puente-Martínez, A., Ubillos-Landa, S., Echeburúa, E. \& Páez-Rovira, D. (2016). Factores de riesgo asociados a la violencia sufrida por la mujer en la pareja: una revisión de metaanálisis y estudios recientes. Anales de Psicología, 32(1), 295-306. http:// dx.doi.org/10.6018/analesps.32.1.189161

Rey-Anacona, C. A. (2009). Maltrato de tipo físico, psicológico, emocional, sexual y económico en el noviazgo: un estudio exploratorio. Acta Colombiana de Psicología, 12(2), 27-36.

Rey-Anacona, C. A. (2013). Prevalencia y tipos de maltrato en el noviazgo en adolescentes y adultos jóvenes. Terapia Psicológica, 31(2), 143-154. http://dx.doi.org/10.4067/S071848082013000200001

Roberts, A. L., Gilman, S. E., Fitzmaurice, G., Decker, M. R. \& Koenen, K. C. (2010). Witness of intimate partner violence in childhood and perpetration of intimate partner violence in adulthood. Epidemiology, 21(6), 809-818. http://dx.doi. org/10.1097/EDE.0b013e3181f39f03

Rodríguez-Domínguez, C., Durán-Segura, M. \& Martínez-Pecino, R. (2018). Ciberagresores en el noviazgo adolescente y su relación con la violencia psicológica, el sexismo y los celos. Health and Addictions, 18(1), 17-27. http://dx.doi.org/10.21134/haajv18i1.329

Rubio-Garay, F., Carrasco, M. A., Amor, P. J. \& López-González, M. A. (2015). Factores asociados a la violencia en el noviazgo entre adolescentes: una revisión crítica. Anuario de Psicología Jurídica, 25(1), 47-56.

Ruiz-Pérez, I., Rodríguez-Barranco, M., Cervilla, J. A. \& Ricci-Cabello, I. (2018). Intimate partner violence and mental disorders: Co-occurrence and gender differences in a large cross-sectional population based study in Spain. Journal of Affective Disorders, 229, 69-78. http://dx.doi.org/10.1016/j.jad.2017.12.032 
Shorey, R. C., Fite, P. J., Cohen, J. R., Stuart G. L. \& Temple, J. R. (2018). The stability of intimate partner violence perpetration from adolescence to emerging adulthood in sexual minorities. Journal of Adolescent Health, 62(6), 747-749. http://dx.doi.org/10.1016/j.jadohealth.2017.11.307

Stovera, C. S., Choib, M. J. \& Mayesa, L. C. (2018). The moderating role of attachment on the association between childhood maltreatment and adolescent dating violence. Children and Youth Services Review, 94, 679-688. http://dx.doi.org/10.1016/j. childyouth.2018.09.011

Sugg, N. (2015). Intimate partner violence prevalence, health consequences and intervention. Medical Clinics of North America, 99(3), 629-649. http://dx.doi.org/10.1016/j.mcna.2015.01.012

Temple, J. R., Choi, H. J., Elmquist, J., Hecht, M., Miller-Day, M., Stuart, G. L., Brem, M. \& Wolford-Clevenger, C. (2016). Psychological abuse, mental health, and acceptance of dating violence among adolescents. Journal of Adolescent Health, 59(2), 197-202. http://dx.doi.org/10.1016/j.jadohealth.2016.03.034

Temple, J. R., Shorey, R. C., Fite, P. J., Stuart, G. L. \& Le, V. D. (2013). Substance use as a longitudinal predictor of the perpetration of teen dating violence. Journal of Youth and Adolescence, 42(4), 596-606. http://dx.doi.org/10.1007/s10964-0129877-1

Tenkorang, E. Y. \& Owusu, A. Y. (2018). A life course understanding of domestic and intimate partner violence in Ghana. Child Abuse \& Neglect, 79, 384-394. http://dx.doi.org/10.1016/j. chiabu.2018.02.027

Van Ouytsel, J., Ponnet, K. \& Walrave, M. (2017). The associations of adolescents' dating violence victimization, well-being and engagement in risk behaviors. Journal of Adolescence, 55, 6671. http://dx.doi.org/10.1016/j.adolescence.2016.12.005
Vink, M., Derks J. M., Hoogendam, J. M., Hillegers, M. \& Kahna, R. S. (2014). Functional differences in emotion processing during adolescence and early adulthood. Neurolmage, 91, 70-76. http://dx.doi.org/10.1016/j.neuroimage.2014.01.035

Vu, N. L., Jouriles, E. N., McDonald, R. \& Rosenfield, D. (2016). Children's exposure to intimate partner violence: A meta-analysis of longitudinal associations with child adjustment problems. Clinical Psychology Review, 46, 25-33. http://dx.doi. org/10.1016/j.cpr.2016.04.003

Wolfe, D. A., Crooks, C. V., Lee, V., Mclntyre-Smith, A. \& Jaffe, P. J. (2003). The effects of children's exposure to domestic violence: A meta-analysis and critique. Clinical Child and Family Psychology Review, 6(3), 171-187. http:dx.doi.org/10.1023/A:1024910416164

Wincentak, K., Connolly, J. \& Card, N. (2017). Teen dating violence: A meta-analytic review of prevalence rates. Psychology of Violence, 7(2), 224-241. http://dx.doi.org/10.1037/a0040194

Yim, I. S. \& Kofman, Y. B. (2018). The psychobiology of stress and intimate partner violence. Psychoneuroendocrinology. http:// dx.doi.org/10.1016/j.psyneuen.2018.08.017

Yu, R., Peplerb, D. J., Van de Bongardt, D., Josephsond, W. I. \& Connolly J. (2018). Internalizing symptoms and dating violence perpetration in adolescence. Journal of Adolescence, 69, 8891. http://dx.doi.org/10.1016/j.adolescence.2018.09.008 